\title{
State Estimation in the Automotive SCR DeNOx Process
}

Zhou, Guofeng; Jørgensen, John Bagterp; Duwig, Christophe; Huusom, Jakob Kjøbsted

Publication date:

2012

Document Version

Publisher's PDF, also known as Version of record

Link back to DTU Orbit

Citation (APA):

Zhou, G., Jørgensen, J. B., Duwig, C., \& Huusom, J. K. (2012). State Estimation in the Automotive SCR DeNOx Process. Poster session presented at 8th IFAC Symposium on Advanced Control of Chemical Processes, Singapore, Singapore.

\section{General rights}

Copyright and moral rights for the publications made accessible in the public portal are retained by the authors and/or other copyright owners and it is a condition of accessing publications that users recognise and abide by the legal requirements associated with these rights.

- Users may download and print one copy of any publication from the public portal for the purpose of private study or research.

- You may not further distribute the material or use it for any profit-making activity or commercial gain

- You may freely distribute the URL identifying the publication in the public portal

If you believe that this document breaches copyright please contact us providing details, and we will remove access to the work immediately and investigate your claim 


\section{State Estimation in the Automobile SCR DeNO Process}

Guofeng Zhou, John B. J ørgensen, Christophe Duwig \& Jakob K. Huusom

ADCHEM 2012, Singapore

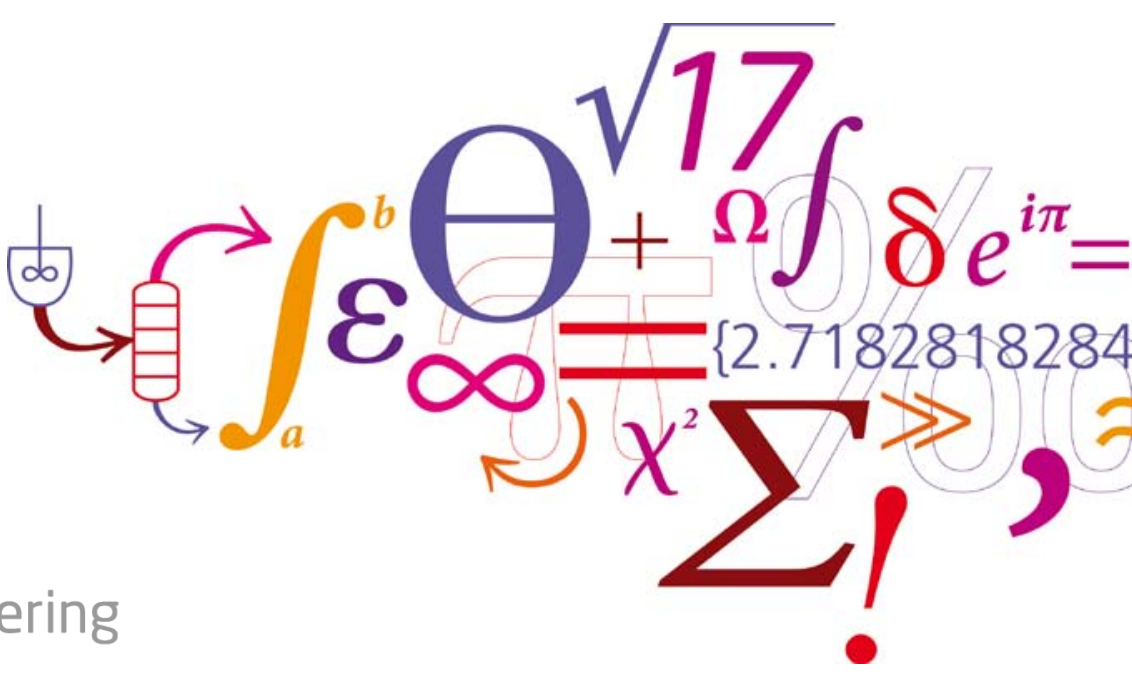




\section{Outline}

\section{$>$ Motivation}

$>$ Modeling the SCR System

$>$ State Estimation

$>$ Simulation Results

Conclusions 


\section{Motivation}

Air quality, especially in urban areas is challenged by exhaust gasses from road vehicles.

Pollutants such as $\mathrm{CO}, \mathrm{NO}_{\mathrm{x}}$ and particular matter from vehicles are harmful for the human respiratory system. $\mathrm{NO}_{\mathrm{x}}$ further contribute to acid rain, formation of $\mathrm{O}_{3}$ and smog conditions.

Diesel engine fume has been elevated to known carcinogen level by W.H.O. on June 12, 2012.

It is estimated that $30 \%$ of the $\mathrm{NO}_{\mathrm{x}}$ and $65 \%$ of the particular matter emissions from road vehicles are due to heavy duty diesel vehicles even thou these only comprise only $2 \%$ of the total on road population. 


\section{Motivation}

Worldwide, legislations are made to gradually reduce emission limits from diesel cars and trucks to counteract these problems.

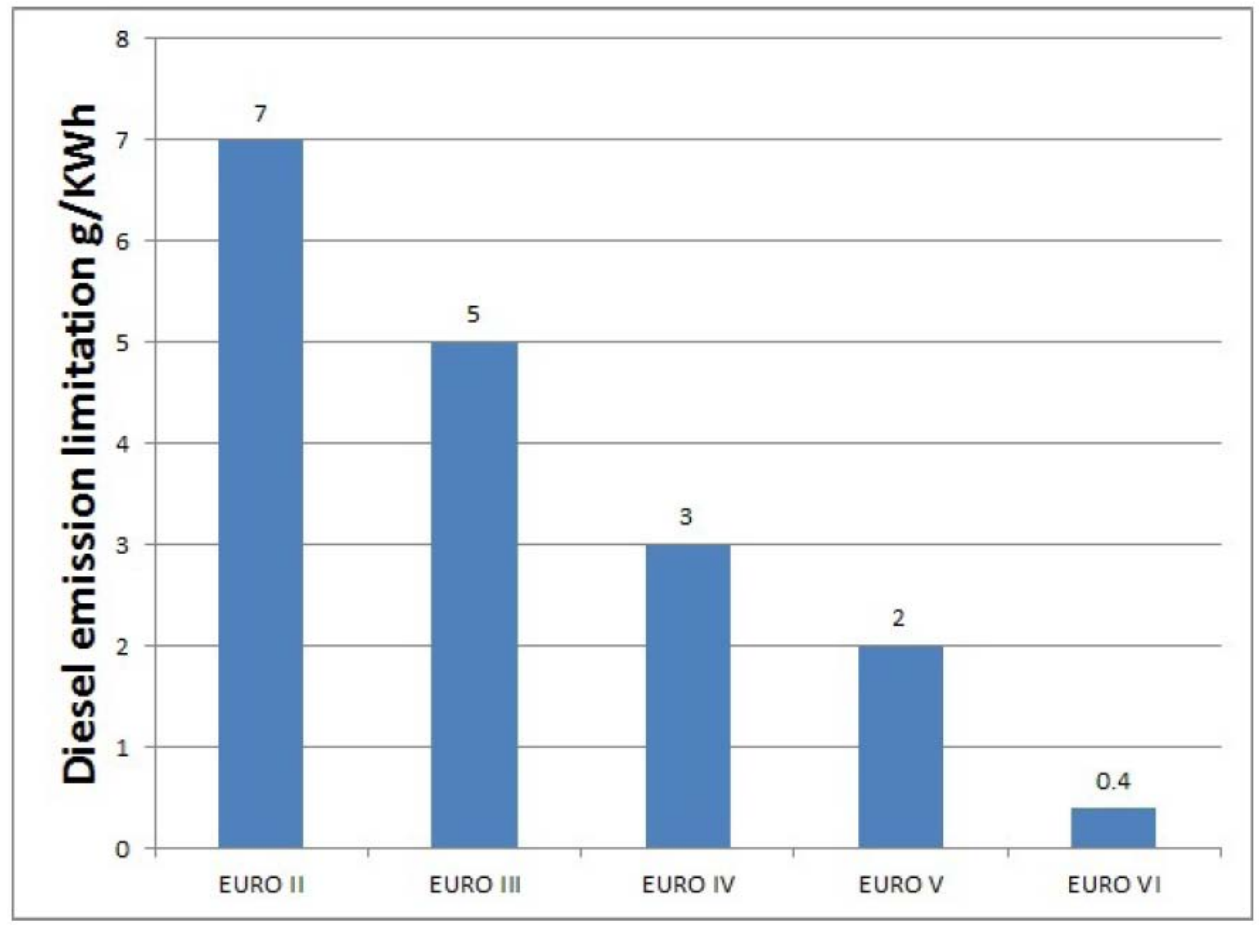

Efficient exhaust gas after treatment systems exists, but as the limits are reduced more intelligent systems are required. 


\section{Diesel engines exhaust gas cleaning system}

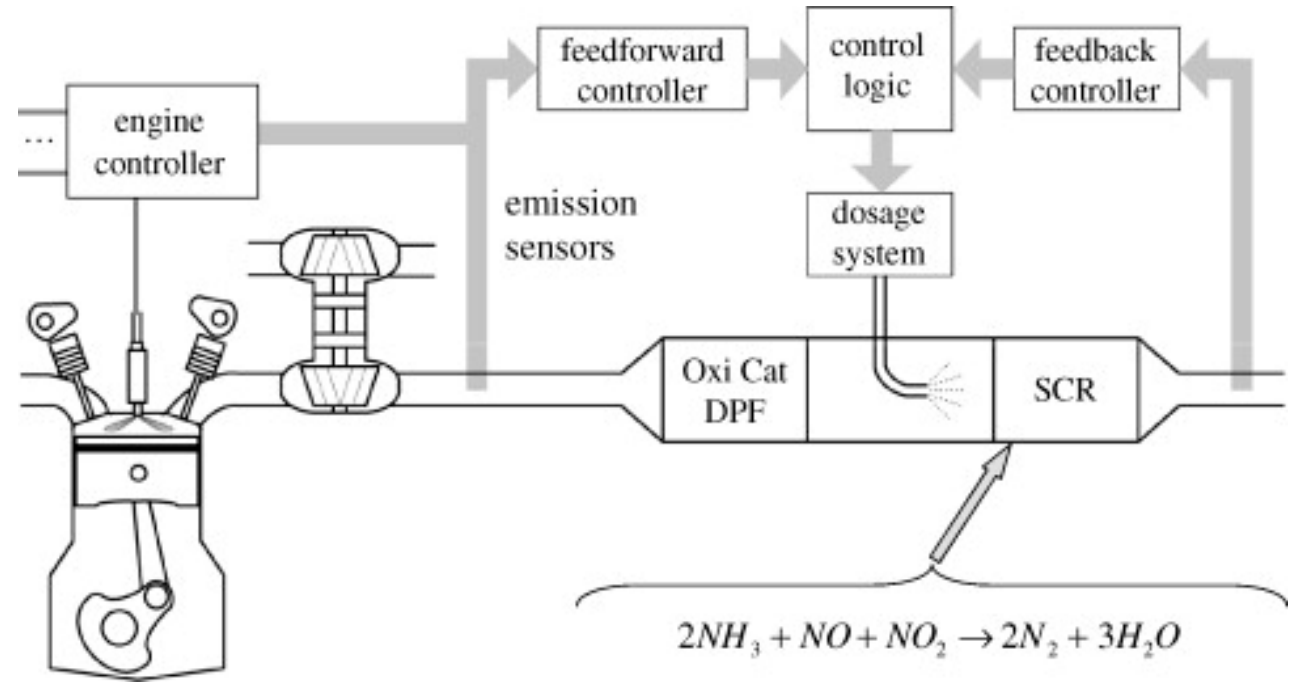

Guzzella 2009, Annual Reviews in Control

\section{Control objectives}

Over a full driving cycle an efficient exhaust gas cleaning system will

- Minimize emissions of nitrogen oxides

- Avoid ammonia slip from the catalyst 


\section{Modeling the SCR system}

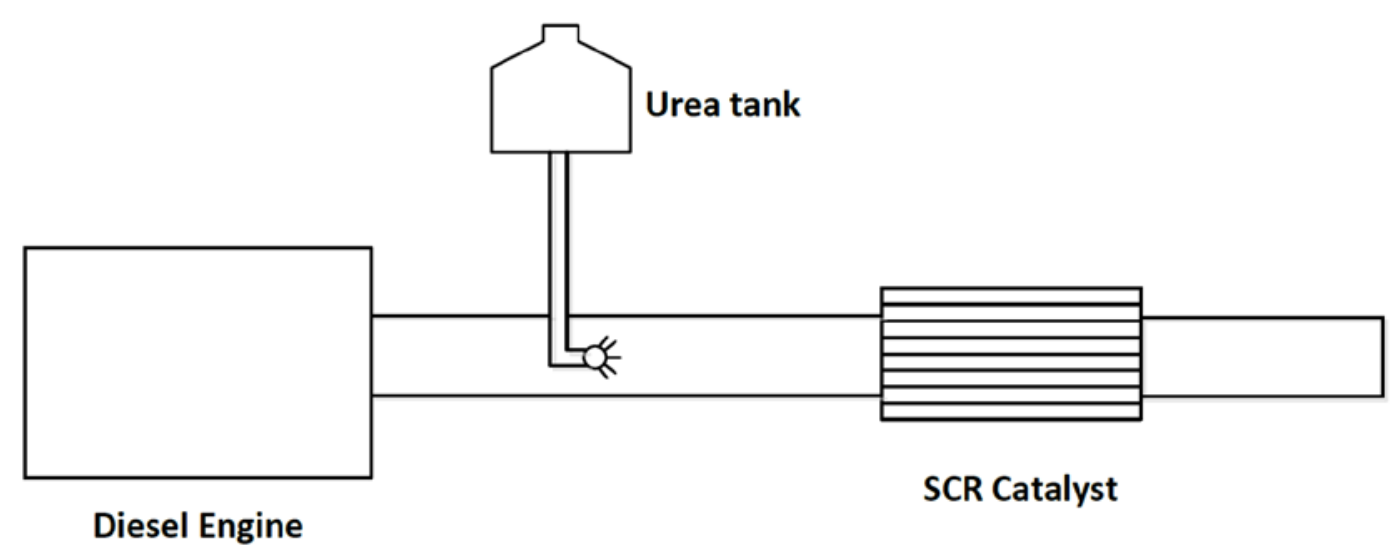

\section{Modeling Assumptions:}

- The flow in the SCR can be modeled as a CSTR

- Energy balances can be neglected and the system temperature equals the flue gas

- No deactivation of the catalyst

- All chemical reactions temperature dependent assuming an Arrhenius expression for the kinetics constants

- Instantaneous conversion of urea to ammonia 


\section{Modeling the SCR system - Chemistry}

Adsorption and desorption of ammonia on the catalyst surface

$$
\begin{aligned}
\mathrm{NH}_{3}+(*) \rightleftharpoons N H_{3}^{*} & r_{a d s}=k_{a d s} C_{N H_{3}}\left(1-\theta_{N H_{3}}\right) \\
& r_{d e s}=k_{d e s} \theta_{N H_{3}}
\end{aligned}
$$

Reduction of $\mathrm{NO}_{\mathbf{x}}$ (standard SCR reaction)

$4 \mathrm{NH}_{3}^{*}+4 \mathrm{NO}+\mathrm{O}_{2} \rightarrow 4 \mathrm{~N}_{2}+6 \mathrm{H}_{2} \mathrm{O}$

$$
r_{r e d 1}=k_{r e d 1} C_{N O} \theta_{N H_{3}}
$$

Reduction of $\mathbf{N O}_{\mathbf{x}}$ ( fast SCR reaction)

$2 \mathrm{NH}_{3}^{*}+\mathrm{NO}+\mathrm{NO}_{2} \rightarrow 2 \mathrm{~N}_{2}+3 \mathrm{H}_{2} \mathrm{O}$

$$
r_{\text {red } 2}=k_{r e d 2} C_{N O} C_{N_{2}} \theta_{N_{3}}
$$

Ammonia oxidation

$4 \mathrm{NH}_{3}^{*}+3 \mathrm{O}_{2} \rightarrow 2 \mathrm{~N}_{2}+6 \mathrm{H}_{2} \mathrm{O}$

$$
r_{o x}=k_{O x} C_{O_{2}} \theta_{N_{3}}
$$




\section{Modeling the SCR system}

\section{Component balances over the SCR system}

$$
\begin{aligned}
\dot{C}_{N O} & =F / V\left(C_{N O, i n}-C_{N O}\right)-4 \Theta k_{r e d 1} C_{N O} \theta_{N H_{3}}-\Theta k_{r e d 2} C_{N O} C_{N O_{2}} \theta_{N H_{3}} \\
\dot{C}_{N O_{2}} & =F / V\left(C_{N O_{2}, i n}-C_{N O_{2}}\right)-\Theta k_{r e d 2} C_{N O} C_{N O_{2}} \theta_{N H_{3}} \\
\dot{C}_{N H_{3}} & =F / V\left(C_{N H_{3}, i n}-C_{N H_{3}}\right)+\Theta k_{d e s} \theta_{N H_{3}}-\Theta k_{a d s} C_{N H_{3}}\left(1-\theta_{N H_{3}}\right) \\
\dot{\theta}_{N H_{3}} & =k_{a d s} C_{N H_{3}}\left(1-\theta_{N H_{3}}\right)-k_{d e s} \theta_{N H_{3}}-4 k_{r e d 1} C_{N O} \theta_{N H_{3}}-2 k_{r e d 2} C_{N O} C_{N O_{2}} \theta_{N H_{3}}-2 k_{o x} C_{O_{2}} \theta_{N H_{3}}
\end{aligned}
$$

The model result in the following general system of ODE's

$$
\begin{gathered}
\dot{x}=f(x(t), u(t), d(t)) \\
x=\left[\begin{array}{c}
C_{N O} \\
C_{N O_{2}} \\
C_{N H_{3}} \\
\theta_{N H_{3}}
\end{array}\right], \quad u=C_{N H_{3}, i n}, \quad d=\left[\begin{array}{c}
F \\
T \\
C_{N O, i n} \\
C_{N O_{2}, i n} \\
C_{O_{2}, i n}
\end{array}\right]
\end{gathered}
$$




\section{State Estimation}
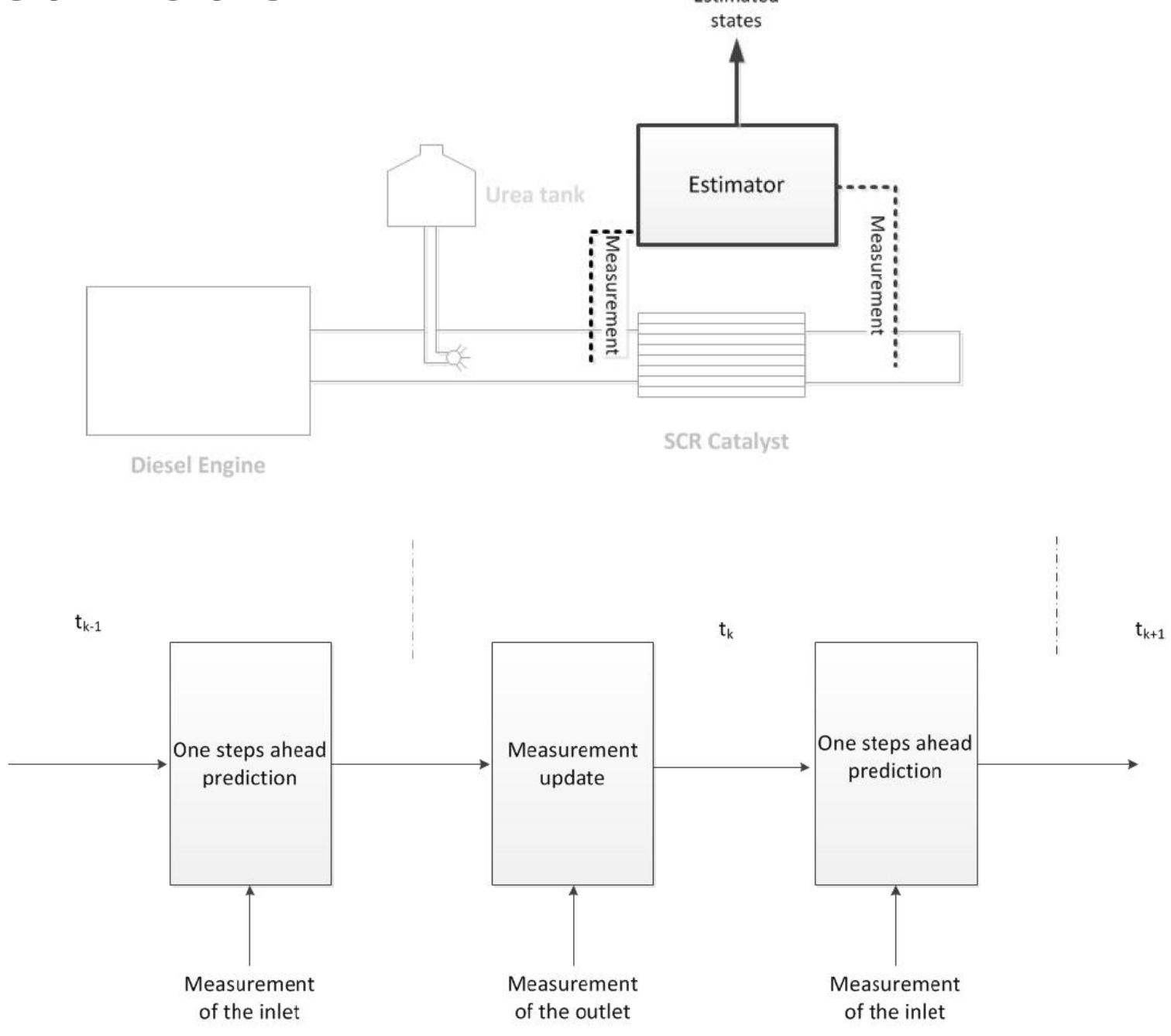


\section{State Estimation - Ordinary Kalman Filter}

Discrete time system description

$$
\begin{aligned}
x_{k+1}^{d} & =A x_{k}^{d}+B u_{k}^{d}+E d_{k}^{d}+w_{k} \\
y_{k} & =C x_{k}+v_{k}
\end{aligned}
$$

Measurement Update

$$
\begin{aligned}
e_{k} & =y_{k}^{d}-C \hat{x}_{k \mid k-1}^{d} \\
K_{f, k} & =P_{k \mid k-1} C^{T}\left[C P_{k \mid k-1} C^{T}+R_{v}\right]^{-1} \\
\hat{x}_{k \mid k}^{d} & =\hat{x}_{k \mid k-1}^{d}+K_{f, k} e_{k} \\
P_{k \mid k} & =P_{k \mid k-1}-K_{f, k}\left[C P_{k \mid k-1} C^{T}+R_{v}\right] K_{f, k}^{T}
\end{aligned}
$$

Time update (one step ahead prediction)

$$
\begin{aligned}
& \hat{x}_{k+1 \mid k}^{d}=A \hat{x}_{k \mid k}^{d}+B u_{k}^{d}+E d_{k}^{d} \\
& P_{k+1 \mid k}=A P_{k \mid k} A^{T}+R_{w}
\end{aligned}
$$




\section{State Estimation - Extended Kalman Filter}

Measurement Update

$$
\begin{aligned}
e_{k} & =y_{k}-C \hat{x}_{k \mid k-1} \\
K_{f, k} & =P_{k \mid k-1} C^{T}\left[C P_{k \mid k-1} C^{T}+R_{v}\right]^{-1} \\
\hat{x}_{k \mid k} & =\hat{x}_{k \mid k-1}+K_{f, k} e_{k} \\
P_{k \mid k} & =P_{k \mid k-1}-K_{f, k}\left[C P_{k \mid k-1} C^{T}+R_{v}\right] K_{f, k}^{T}
\end{aligned}
$$

Time update (one step ahead prediction)

$$
\begin{aligned}
\hat{x}_{k+1 \mid k} & =\hat{x}_{k \mid k}+\int_{t_{k}}^{t_{k+1}} f\left(x(\tau), u_{k}, d_{k}\right) d \tau \\
\bar{A}_{k} & =I+\int_{t_{k}}^{t_{k+1}} \frac{\partial f}{\partial x}\left(x(\tau), u_{k}, d_{k}\right) S_{\hat{x}_{k \mid k}} d \tau \\
P_{k+1 \mid k} & =\bar{A}_{k} P_{k \mid k} \bar{A}_{k}^{T}+R_{w} \\
\dot{S}_{\hat{x}_{k \mid k}} & =\frac{\partial f}{\partial x}\left(x(t), u_{k}, d_{k}\right) S_{\hat{x}_{k \mid k}} \quad \text { ADCHEM 12/07/2012 }
\end{aligned}
$$




\section{Simulation Results \\ I deal model; $\mathrm{NO}, \mathrm{NO}_{2}$ and $\mathrm{NH}_{3}$ measurement}
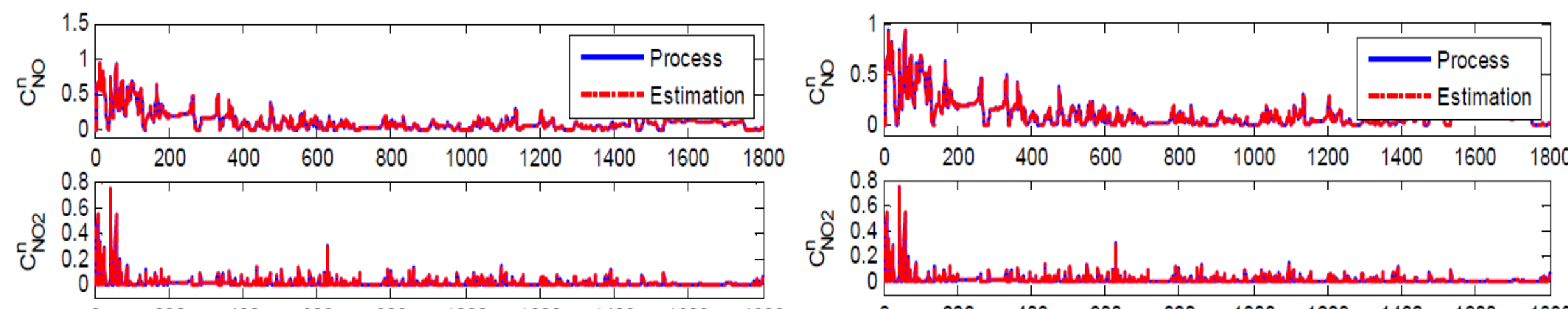

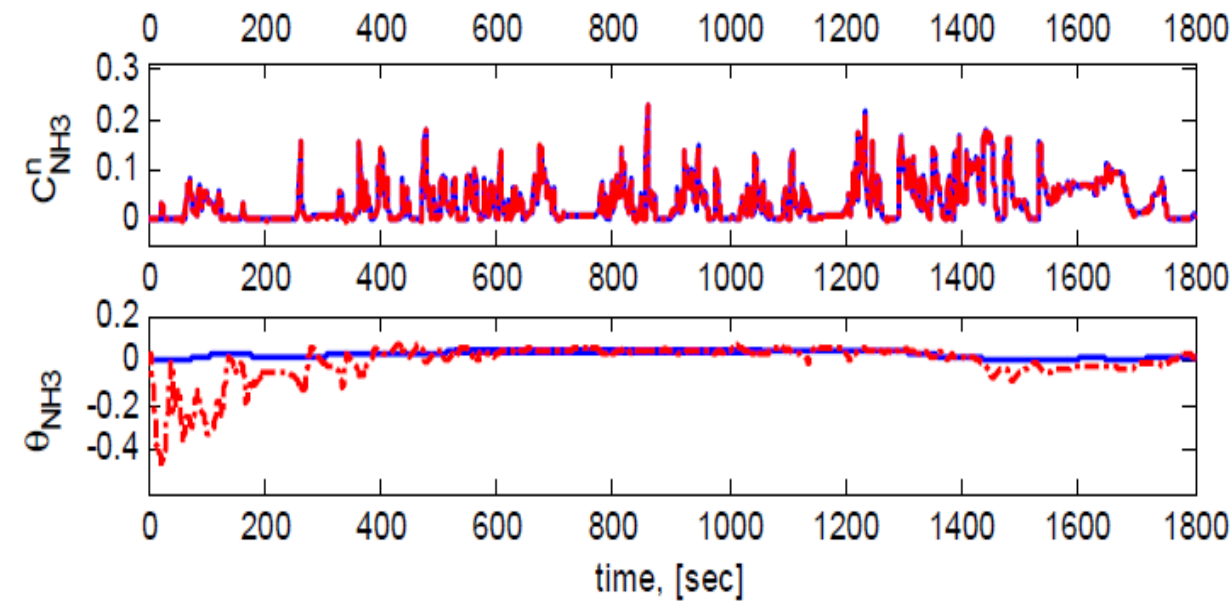

Ordinary Kalman Filter
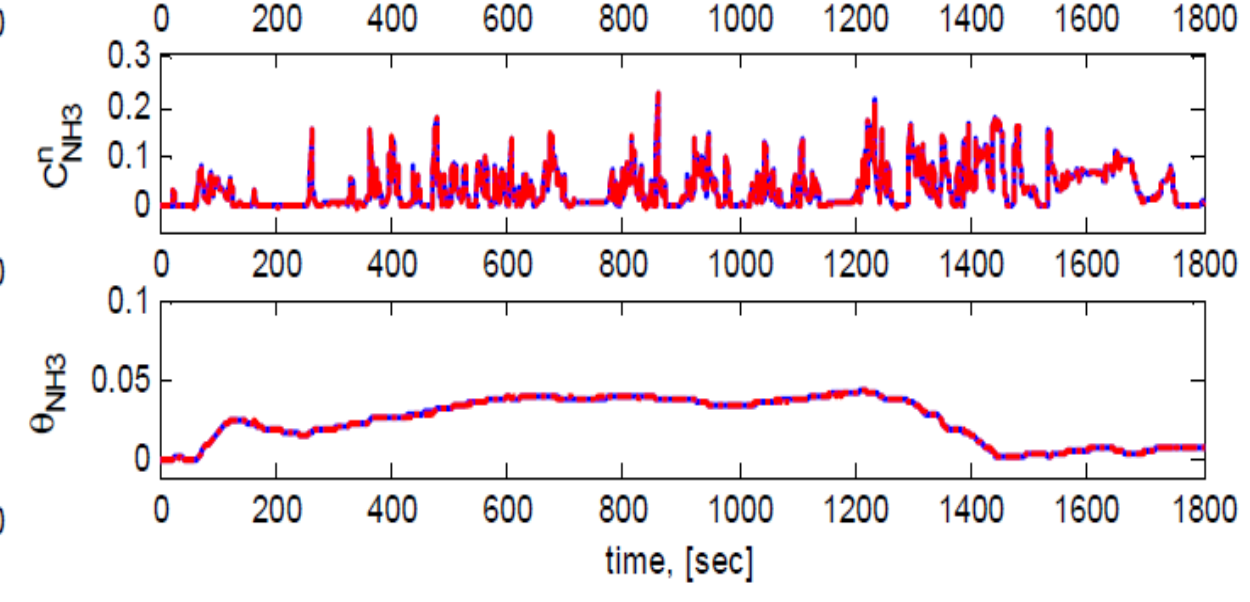

Extended Kalman Filter 


\section{Simulation Results \\ I deal model; $\mathrm{NO}_{x}$ and $\mathrm{NH}_{3}$ measurement}
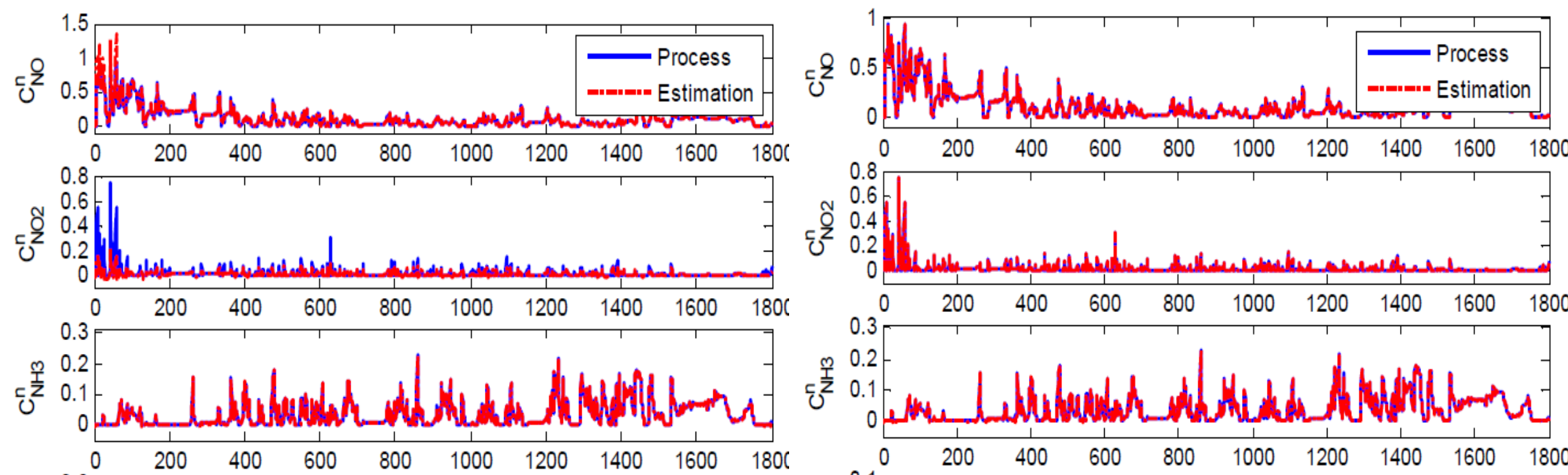

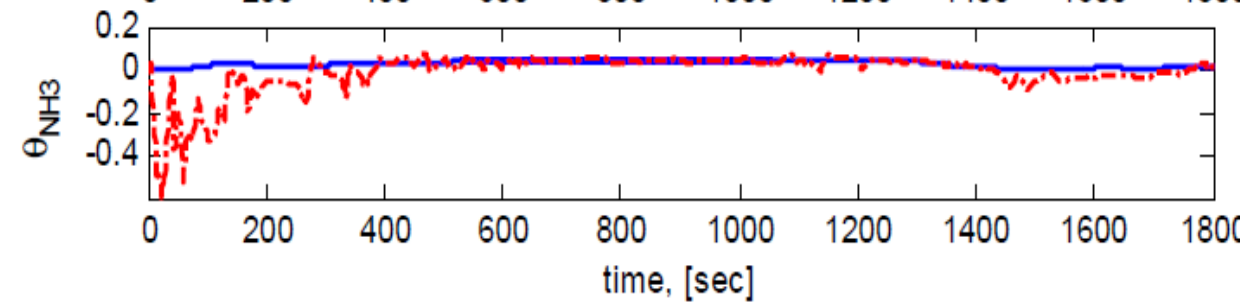

Ordinary Kalman Filter

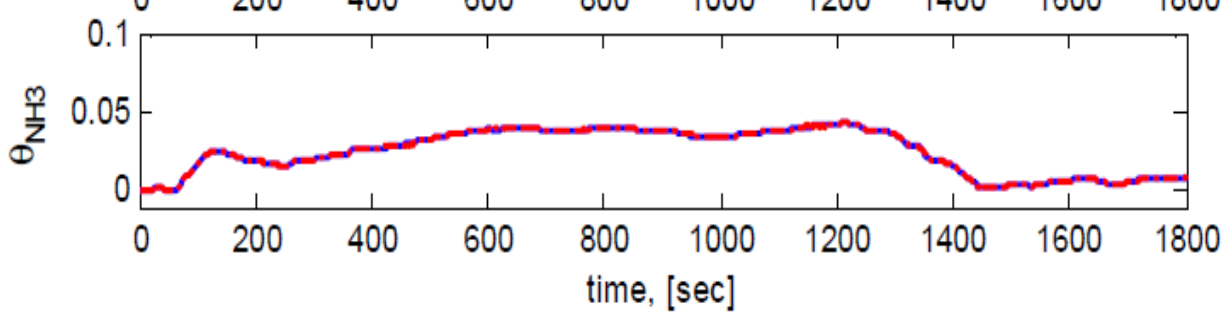

Extended Kalman Filter 


\section{Results so far ...}

We see that the extended Kalman filter clearly outperformed the ordinary formulation for this process, hence it is necessary to include the nonlinear dynamics in the state estimation.

The results so far have been promising, but we also assume perfect model information.

In reality the flow regime is not perfectly well mixed but plug flow with some degree of axial dispersion.

Other assumptions are somewhat questionable too.

- Transport phenomena from the bulk gas to the surface

- Modeling of the catalyst surface temperature 


\section{Conclusions}

A nonlinear filter is necessary to estimate ammonia coverage of the catalyst surface in the SCR unit.

The current model we use has a good level of complexity for fast computations but it lacks some accuracy needed for precise estimates

- Flow

- Surface temperature

- Transport limitations

The proposed filter would be useful in model based control implementations

- Feed forward + simple PI type feedback control

- Model Predictive Control 